\title{
Guidelines for Computing Longitudinal Dynamic Stability Characteristics of a Subsonic Transport
}

\author{
Joseph R. Thompson ${ }^{1}$ \\ University of Alabama at Birmingham, Birmingham, Alabama, 35294 \\ Neal T. Frink ${ }^{2}$ and Patrick C. Murphy ${ }^{3}$ \\ NASA Langley Research Center, Hampton, Virginia, 23696
}

\begin{abstract}
A systematic study is presented to guide the selection of a numerical solution strategy for URANS computation of a subsonic transport configuration undergoing simulated forced oscillation about its pitch axis. Forced oscillation is central to the prevalent wind tunnel methodology for quantifying aircraft dynamic stability derivatives from force and moment coefficients, which is the ultimate goal for the computational simulations. Extensive computations are performed that lead in key insights of the critical numerical parameters affecting solution convergence. A preliminary linear harmonic analysis is included to demonstrate the potential of extracting dynamic stability derivatives from computational solutions.
\end{abstract}

\section{Nomenclature}

$C_{L} \quad=$ lift coefficient

$C_{m}$ or $C M=$ pitching moment coefficient

$c_{\text {ref }} \quad=$ mean aerodynamic reference chord, $=10.98 \mathrm{in}$.

$f$

$=$ sinusoidal oscillation frequency about pitch, roll, or yaw axis, Hz.

$k \quad=$ reduced frequency, $=2 \pi f \cdot\left(c_{r e f} / 2\right) / U_{\infty}$

$\log (r / r 0) \quad=$ order of magnitude drop in solution residual, e.g. -3 means 3 orders magnitude reduction

$M_{\text {USM } 3 D} \quad=$ USM3D freestream Mach number, $=0.2$

$M_{\infty} \quad=$ experimental freestream Mach number, $=0.077$

$n, n+1 \quad=$ indices for numerical time step

$R e_{\text {cref }}=$ Reynolds number based on $c_{\text {ref }}$

rate1

rate 2

$\Delta t$

$\Delta t^{*}$

$U_{\infty}$

$y^{+}$

$\alpha$

$\alpha_{0}$

$\Delta \alpha$

$\delta_{t+1}$

$\delta_{l}$

$=$ primary VGRID "viscous" stretching factor, see Eq. 1

$=$ secondary VGRID "viscous" stretching factor, see Eq. 1

$=$ incremental time step, sec

$=$ characteristic time step, $=\Delta t \bullet U_{\infty} / c_{\text {ref }}$

$=$ freestream velocity, $=86 \mathrm{ft} / \mathrm{sec}$

$=$ turbulence wall coordinate

$=$ angle of attack (static: $\alpha$, deg, dynamic: $\alpha=\alpha_{0}+\Delta \alpha$, deg)

$=$ nominal angle of attack during pitch oscillation, deg.

$=$ incremental angle of pitch oscillation about body axis, deg.

$=$ VGRID "viscous" grid spacing normal to surface at node $i+1$, see Eq. 1, inches

$=$ spacing of first node off of surface $(i=1)$ in "viscous" grid layers, see Eq. 1, inches

\footnotetext{
${ }^{1}$ Graduate Student. Work performed onsite at NASA Langley Research Center.

${ }^{2}$ Aerospace Engineer, Configuration Aerodynamics Branch, Research Directorate, MS 499, Associate Fellow AIAA.

${ }^{3}$ Aerospace Engineer, Dynamic Systems \& Control Branch, Research Directorate, MS 308, Associate Fellow AIAA.
} 
$\underline{\text { Key Acronyms }}$

$\begin{array}{ll}\text { GTM } & \text { Generic Transport Model } \\ \text { SA } & =\text { Spalart-Allmaras one equation turbulence model } \\ \text { SST } & =\text { Menter's Shear-Stress Transport two equation turbulence model } \\ \text { URANS } & =\text { Unsteady Reynolds-Averaged Navier-Stokes } \\ 14 X 22 & =\text { NASA Langley 14- by 22-Foot Low-Speed Wind Tunnel }\end{array}$

\section{Introduction}

The NASA Aviation Safety Program is sponsoring a multi-year effort through the Integrated Resilient Aircraft Control (IRAC) project to improve the survivability of civil transport aircraft that encounter critical loss-of-control (LOC) flight upset conditions. IRAC's intended goal is the creation of validated, multidisciplinary integrated aircraft control design tools and techniques that can be used to enable safe flight in the midst of adverse flight conditions (i.e. faults, damage, icing, and/or upset) by addressing aircraft stability and control (S\&C) parameters [1].

In the late 1990's the US Government along with Industry formulated a partnership to address and develop plans to reduce aviation accidents and loss of life. That partnership developed into the Commercial Aviation Safety Team (CAST). During their investigation of the data it was learned that the highest contributor to loss of life were LOC events as can been seen in Fig. 1 from data gathered by the National Safety Transportation Board (NSTB) [2].

A series of intervention strategies were developed to reduce the occurrence of LOC accidents, which can arise from a variety of causes. From the initial analysis and developmental phase it was determined that the current simulation technology was inadequate to train and prepare pilots for realistic conditions outside of the normal flight envelope (i.e. stall, post-stall and upset conditions). Traditionally there have been three methods of choice used in analyzing S\&C characteristics. Flight-testing the actual aircraft, would be the obvious first and most accurate, though not necessarily, most economical choice [3,4]. This method is prohibitive not only because of the cost involved, but also

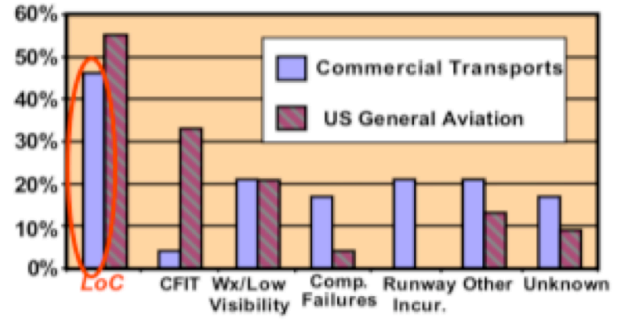

Figure 1. Fatal accident distribution for commercial transports and general aviation. Source NSTB database 1990-96 because of the inherent danger posed to the flight crew as a result of an inadequate escape mechanism in transport aircraft for cockpit members. Wind tunnel testing on scaled models is the second method of choice, but it too has large cost and time requirements [3]. In addition to cost and time concerns, other factors such as Reynolds-number scaling, blockages and interference to the flow caused by the model support system prevent proper modeling of the full scale behavior and contribute to limiting the use of this technique. Incorporating the use of data sheets, linear aerodynamic theory, and empirical relations in the third method can be effective in typical flight maneuvers but is very limited in its ability to predict flight dynamics at the outer edges of the flight envelope in stall, post-stall or upset conditions [3].

Figure 2 portrays a graphical representation of the limitations of the current state of the art in predicting flight dynamics in the outer edges of the flight envelope. The chaotic red line, representing "black box" data from an aircraft experiencing a LOC accident, reveals a flight trajectory well outside of the available wind tunnel data. Two of the main reasons behind the limited flight data are: 1) the current data sets meet the minimum requirements for simulator certification, which do not typically explore beyond normal flight conditions and 2) flight and wind-tunnel test do not usually provide aerodynamic measurements of upset conditions. To bridge this data gap, Computational Fluid Dynamics (CFD) is being utilized to analyze flight dynamics in the outer reaches of the flight envelope.

This study contributes to the Integrated Dynamics \&

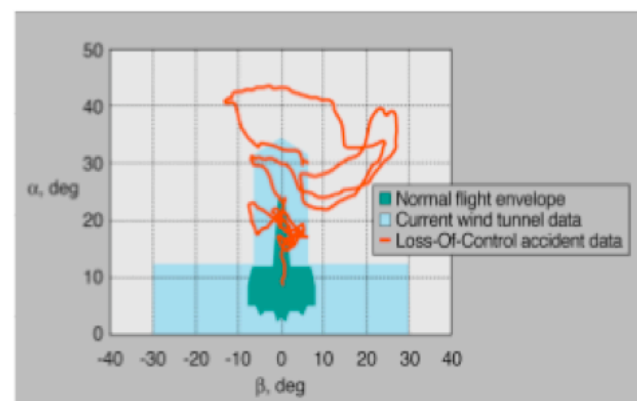

Figure 2. Graphical representation of available aerodynamic data for flight envelope undergoing LOC [2].

Flight Controls (IDFC) subproject within IRAC. Within the modeling components IDFC, the goal is to develop reliable computational tools for predicting and analyzing stability and control characteristics of aircraft in damaged and upset flight conditions. A prior study reported in Ref. [5] advances this objective by assessing the utility of two 
unstructured Navier-Stokes flow solvers for capturing the degradation in static stability and aerodynamic performance of a subsonic transport due to airframe damage. The present work delves into the complex realm of dynamic stability in upset flight conditions. The focus is to assess the applicability of CFD modeling for dynamic aircraft motions on the post-stall aerodynamics that are characteristic of LOC flight. The following study has been initiated to develop the practices for computing the aerodynamic and stability characteristics of an aircraft undergoing periodic oscillation about a post-stall angle of attack using the tetrahedral-based USM3D unsteady Reynolds Averaged Navier-Stokes (URANS) flow solver. To be assessed are 1) the accuracy and efficiency of the NASA USM3D flow solver for predicting the hysteresis behavior of force and moment coefficients for a subsonic transport model undergoing pitch oscillation, and 2) the sensitivities of USM3D input parameters affecting timeaccuracy of the aircraft in dynamic motion which will contribute to the development of practical guidelines for applying USM3D for future dynamic S\&C problems. This effort supplements and reinforces the findings from a similar study performed on unmanned military air vehicle [6]. While it is recognized that URANS methodology may not be suitable for massively separated flows, the intent is to lay a solid numerical foundation for future studies, which will include more advanced turbulence models.

\section{Geometry and Experiment}

The IRAC focus configuration (Fig. 3) is a representative twin-engine commercial transport configuration called the Generic Transport Model (GTM) that has received extensive static and dynamic force and moment testing in the NASA Langley 14- by 22-Foot Subsonic Tunnel (14X22) [7-9]. Conventional forced-oscillation tests conducted in 2001 and 2003 were expanded upon in 2007 and 2008 to include a broader range of oscillation frequencies that would allow identification of unsteady aerodynamic mathematical models. The model span is 82.2 inches, and the mean aerodynamic reference chord, $c_{\text {ref }}$, is 10.98 inches. The tests were conducted at sea level atmospheric conditions with a dynamic pressure of $10 \mathrm{lb} / \mathrm{ft}^{2}$, corresponding to a freestream velocity $U_{\infty}=86 \mathrm{ft} / \mathrm{sec}$, a Mach number $M_{\infty}=0.077$ and chord Reynolds number $R e_{\text {cref }}=0.54 \times 10^{6}$. Boundary layer transition strips of width 0.1 inch and number 36 grit were applied at $10 \%$ chord on the upper and lower surfaces of the wing, horizontal and vertical tails. A 0.1-inch wide ring of number 36-grit was applied 1 inch aft of the apex of the nose (measured longitudinally). This gritting pattern was chosen to maintain commonality with previously tested configurations. At the time of this writing it is unclear if this technique was effective in tripping the flow (there was no verification performed, and with the exception of the nose grit, this particular tripping technique differs from well established low-speed practices, e.g. Braslow [10]). However, these are the limitations of dynamic wind tunnel testing that might be expanded by CFD application.

Forced oscillation tests were conducted for the baseline and damaged GTM geometries. The configurations were oscillated over a nominal range of roll, pitch, and yaw body angles in a sinusoidal fashion at frequencies and amplitudes that enable the identification of both conventional aerodynamic characteristics and advanced mathematical models for rigid-body aerodynamics in nonlinear unsteady flight regimes. Oscillation tests were conducted individually in each axis to acquire aerodynamic damping characteristics. For this study sinusoidal pitch oscillation of $\Delta \alpha= \pm 5$ deg was implemented about $\alpha_{0}=24 \mathrm{deg}$ with frequencies $f=0.43,0.12$, and $0.05 \mathrm{~Hz}$, which correspond to reduced frequencies $k=2 \pi f \cdot\left(c_{r e f} / 2\right) / U_{\infty}=0.01438,0.00401$, and 0.00167 , respectively.

\section{Computational Method}

The cell-centered computations are produced from the USM3D Navier-Stokes flow solver [11] that is part of the NASA Tetrahedral Unstructured Software System (TetrUSS) [12]. USM3D is a parallelized tetrahedral cellcentered, finite volume Navier-Stokes flow solver. The term "cell centered" means that the finite volume flow solution is solved at the centroid of each tetrahedral cell. Inviscid flux quantities are computed across each tetrahedral cell face using various upwind schemes. Spatial discretization is accomplished by a novel reconstruction process, based on an analytical formulation for computing solution gradients within tetrahedral cells. The solution is advanced in time [13] by a $2^{\text {nd }}$-order "physical" time step scheme, a $2^{\text {nd }}$-order "dual" time step scheme, or to a steady-state condition by an implicit backward-Euler scheme. Several turbulence models are available: the SpalartAllmaras (SA) one-equation model, the two-equation Jones and Launder $k-\varepsilon$ turbulence model, the Menter Shear Stress Transport (SST) two-equation model, and the nonlinear Algebraic Reynolds Stress Models of Girimaji and Shih/Zhu/Lumley. Detached Eddy Simulation has been implemented in all of the turbulence models. A capability to trip the flow at specified locations on aerodynamic surfaces has been implemented for the $k$ - $\varepsilon$ turbulence model. USM3D has capabilities for dynamic grid motion, which is being utilized in the current study. 


\section{Grid Generation}

Half-span tetrahedral grids of nominally 6-, 12-, and 24- million cells were generated using the unstructured tetrahedral grid generation codes GridTool and VGRID, which are part of the NASA TetrUSS system. The surface triangulation for the 6-million cell grid is shown in Fig. 4. A developmental version of VGRID [14] was used for the present study. Thin-layer tetrahedral grids were generated to meet requirements for cell-centered computations from the USM3D flow solver. The near-wall spacing prescribed to achieve a turbulent wall coordinate $y^{+}$of 0.75 in the tetrahedral cell center near the mid-chord of the $c_{r e f}$. Since the VGRID Advancing Layers Method [15] marches nodes away from the surface (which are subsequently connected to form tetrahedral cells), then an initial VGRID "viscous" spacing corresponding to a $y^{+}=3$ of the first node was prescribed in order to achieve the $y^{+}=0.75$ at the first cell centroid. For the GTM at the wind tunnel test conditions, the required initial first-node wall spacing is $\delta_{1}=0.001239$ inches, and stretching factors of ratel $=0.15$ and rate $2=0.02$, where the nodal spacing layers are defined by the Eq. (1).

$$
\delta_{i+1}=\delta_{I}\left[1+\operatorname{ratel}(1+\text { rate } 2)^{i}\right]^{i}
$$

This spacing distribution results in approximately 54 tetrahedral cells (18 nodes) across the boundary layer at the mid-chord of the $c_{r e f}$. The triangular surface faces were stretched as high as a 10-to-1 ratio along the leading edges of the wing and tails.

\section{Characterization of GTM Aerodynamics}

The character of the static lift and pitching moment polar is presented in Fig. 5. The 14X22 static wind tunnel data [7] extends up to 85 deg angle of attack. Computed results are included at angles of attack of 4, 10, 14, 20, 24, 35 , and 40 degrees on half-span grids of 6-, 12-, and 24-million cells. The static USM3D solutions are computed as time-accurate URANS flow solutions assuming fully turbulent flow with the SA and SST turbulence models at a characteristic time step of $\Delta t^{*}=\Delta t \cdot U_{\infty} / c_{\text {ref }}=0.02$ for 1500 time steps. An assumption is made that the wind tunnel sting and walls can be ignored in these calculations.

Sensitivity to grid density is portrayed in Fig. 5 for half-span grids of 6-, 12-, and 24-million cells using the SA turbulence model. Both the lift and pitching moment coefficients in Fig. 5 indicate a break in slope around $\alpha=10$ deg that is indicative of a wing-stall onset. Lift continues to increase up to a maximum around $\alpha=35$ to $40 \mathrm{deg}$, beyond which the aircraft is in deep stall. The pitching moment continues to lose pitch up authority with increasing angle of attack. The computational solutions suggest that the effect of grid density is minimal relative to their correlations with the wind tunnel data with the exception of $\alpha=14 \mathrm{deg}$, where the 6-million cell grid encounters significant differences relative to the 12- and 24-million cell grids. The computations generally over-predict the lift and under-predict the pitching moment in the post-stall region, which is an expected result due to excessive levels of turbulent viscosity produced at those conditions by the SA turbulence model.

The impact of turbulence model is addressed in Fig. 6 for the 6-million cell grid. The dominant effect is in the stall region beyond $\alpha=10 \mathrm{deg}$. Predicted lift for the SST model is significantly below that of the SA model and experimental data over angle of attack range from 14 to 24 degrees. Significant differences persist at the higher angles of attack with the SST model correlating best at $\alpha=40 \mathrm{deg}$. The SST model is known to produce lower levels of turbulent viscosity than the SA model for massively separated thereby allowing disturbances to propagate more readily to the wing upper surface.

The focus of the present study is on establishing guidelines for computing dynamic S\&C characteristics by mimicking the experimental approach of imposing a periodic oscillation about a nominal angle of attack. Results from the dynamic 14X22 wind tunnel data [9] are presented in Fig. 7 for $\alpha_{0}=24$ deg with $\Delta \alpha=+/-5$ deg of pitch oscillation. The pitching moment coefficients are plotted relative the oscillating body angle, $\Delta \alpha$. Both raw and mean (averaged) experimental data are presented over 40 pitch cycles for oscillation frequencies of $0.43,0.12$, and 0.05 $\mathrm{Hz}$. The scatter of the raw data (plotted in light gray) reflects the difficult nature of experimental dynamic testing where both the aerodynamic behaviors of interest as well as systematic and random measurement errors are present. This data is averaged to create the "mean" wind tunnel data that will be used for comparisons in the computational study. 


\section{Harmonic Analysis and Model Identification}

Conventional flight dynamics simulations incorporate the aerodynamic model by assuming the aerodynamic forces and moments can be approximated by a series expansion in terms of aircraft states and controls. The terms in the expansion are the stability and control derivatives. A large database of knowledge has been built around these derivatives providing substantial insights for analysis and design. Several experimental methods can be used to obtain derivatives with respect to rates (damping) but the conventional method is to use forced-oscillation tests. Conventional analysis of longitudinal forced-oscillation data assumes that the aerodynamic coefficient is a linear function of angle-of-attack, pitching velocity, and their rates. This approach [16] produces in-phase and out-of-phase coefficients, $\bar{C}_{a_{\alpha}}$ and $\bar{C}_{a_{q}}$ respectively, where subscript " $a$ " represents either normal force, $C_{N}$, or pitching moment, $C_{m}$. A method of harmonic analysis [17] can be applied to the measured aerodynamic forces and moments to allow estimation of the in-phase and out-of-phase coefficients. For harmonic analysis a mathematical model is postulated as

$$
C_{a}(t)=A_{0}+\sum_{j=1}^{r} A_{j} \cos (j \omega t)+\sum_{j=1}^{r} B_{j} \sin (j \omega t) \quad a=N \text { or } m
$$

where $A_{0}, A_{j}$, and $B_{j}$ are the Fourier coefficients. For a model with linear aerodynamics, the in-phase and out-ofphase components of $C_{a}(t)$ can be expressed in terms of the $1^{\text {st }}$ harmonic Fourier coefficients as

$$
\begin{array}{ll}
\bar{C}_{a_{\alpha}}=\frac{B_{1}}{\alpha_{A}}=C_{a_{\alpha}}-k^{2} C_{a_{\dot{q}}} & a=N \text { or } m \\
\bar{C}_{a_{q}}=\frac{A_{1}}{k \alpha_{A}}=C_{a_{q}}+C_{a_{\dot{\alpha}}} & a=N \text { or } m
\end{array}
$$

Also shown in Eqs. (3-4), are the commonly expressed relationships of these coefficients to the steady flow damping terms and acceleration terms. When an aircraft is operating at low angles-of-attack or in linear and steady aerodynamic flight regimes the out-of-phase component is a good approximation for the damping. This formulation works well with the series expansions used in flight simulations. However, at high angles-of-attack or regions where separated flows dominate, the acceleration terms can become important and demonstrate strong frequency dependence. Under these conditions the nonlinear unsteady aerodynamic behaviors are not well captured by this term alone and more advanced models are required.

Although the conventional model structure has limitations, substantial insights can be gained by comparing the in-phase and out-of-phase components derived from wind tunnel tests against that obtained from CFD calculations. The first harmonic is an important term even under conditions where higher order terms might be required. Due to the orthogonal nature of the model terms the first harmonic is not changed even when higher order terms are required to model nonlinear aerodynamic behavior. Also, the first harmonic will demonstrate frequency dependence in the presence of unsteady aerodynamics. Thus two key aspects of the aerodynamic response can be captured by comparing the aerodynamic force and moment time histories and the $1^{\text {st }}$ harmonic coefficients between wind tunnel and CFD results: (1) time history comparisons demonstrate that full nonlinear aerodynamic responses have been captured, and (2) the $1^{\text {st }}$ harmonic coefficients demonstrate the levels of damping and unsteady aerodynamics if present.

\section{Approach to Numerical Guideline Study}

The primary goal for this paper is to establish guidelines for applying USM3D toward computing reasonably accurate dynamic force and moment characteristics on the GTM aircraft with as little computer resource as possible. An approach similar to that outlined in Ref. [6] is applied to explore the relationship between the numbers of time steps and subiterations of the $2^{\text {nd }}$-order time step scheme. The bulk of this work will focus on understanding the relationships between numbers of time steps per oscillation cycle and subiterative convergence between each time step to establish application guidelines for computing dynamic stability characteristics for a spectrum of cases.

The prevalent experimental methodology for analysis of dynamic stability characteristics of an aircraft calls for extracting the stability derivatives from the force and moment coefficients as the aircraft undergoes a sinusoidal motion about an axis, as illustrated in left image of Fig. 8. A similar approach is mimicked in the present work for longitudinal pitch oscillation.

The strategy from [6] incorporates visual correlation to understand the relationship between the number of time steps per oscillation cycle and subiterative convergence at a time step representative of difficult convergence. As illustrated in Fig. 8, a set position within the pitch oscillation cycle (in this case at -3 deg downswing) is selected to 
monitor the correlation between the pitching moment coefficient and its subiterative convergence between the accompanying time step $n$ and $n+1$. The middle plot in Fig. 8 depicts a dynamic pitching moment coefficient, $C_{m}$, that exhibits hysteresis as the GTM oscillates $+/-5$ deg about its pitch axis at $\alpha_{0}=24$ degrees. (The black dashed line is "mean" wind tunnel data from [9].) The arrow leaning to the right denotes the upswing, and the arrow leaning to the left denotes the downswing. The right-hand plot in Fig. 8 conveys the corresponding order-of-magnitude convergence in the USM3D solution residual drop $(\log (r / r 0))$ highlighted in red, and $C_{m}$ highlighted in blue. In this example, the solution residual has dropped over 3 orders of magnitude and the $C_{m}$ is converged after 100 subiterations using 360 time steps per pitch oscillation cycle

This study is organized by first establishing a set of "well converged" reference solutions. These solutions will be generated by applying $2^{\text {nd }}$ order "physical" time stepping in USM3D with the Spalart-Allmaras (USM3D/SA) turbulence model in a manner that balances the numbers of time steps per pitch oscillation with the number of subiterations between time steps, to maintain a fixed number of total iterations. A sufficiently large number of subiterations will be used to insure a well-converged solution between time steps. Next, reducing the numbers of subiterations for a selected oscillation frequency and monitoring its subiterative convergence and resulting departure from the reference solution will identify an efficient solution strategy. Once a successful strategy is determined, it will then be tested with other pitch frequencies, the SST turbulence model, a larger pitch amplitude of $\Delta \alpha=+/-10$ deg, and for grid sensitivities.

\section{Results and Discussion}

The selected GTM test condition is a nominal post-stall $\alpha_{0}=24$ deg with a pitch oscillation of $\Delta \alpha=+/-5$ deg at frequencies of $f=0.43,0.12$, and $0.05 \mathrm{~Hz}$ that correspond to reduced frequencies $k=0.01438,0.00401$, and 0.00167 , respectively. The flow conditions are $M_{U S M 3 D}=0.2$ and $R e_{\text {cref }}=0.54 \times 10^{6}$. The USM3D results were generated at a slightly higher Mach number than the wind tunnel freestream $M_{\infty}=0.077$ to improve the numerical convergence with the assumption that compressibility effects are negligible. Fully turbulent flow is assumed for all solutions. The flow computations were performed with Roe's Flux Difference Splitting [18] and no flux limiter.

\section{A. Establishing "Well Converged" Reference Solutions}

Table 1 shows the matrix of computational runs used to generate "well converged" USM3D solutions using the SA turbulence model at pitch frequencies of $0.43,0.12$, and $0.05 \mathrm{~Hz}$. Time steps were chosen to yield 360,720 , and 1440 steps per pitch cycle. Corresponding levels of subiteration between time steps, 100,50 , and 25 , respectively, were defined to yield a total of 36,000 solution iterations over each pitch cycle. The cells highlighted in blue will be used in the next section in search of more efficient solution strategy.

Figure 9 depicts plots of sub-iterative convergence of solution residuals (red) and pitching moment coefficient (CM) (blue) between time steps $n$ and $n+1$ near the downswing $\Delta \alpha=-3.5^{\circ}$ $\left(\alpha=20.5^{\circ}\right)$ for the cases in Table 1. The order-of-magnitude drop of the solution residual is denoted on the left axis, and CM is on the right axis. The axis increments of $\mathrm{CM}$ are held constant between plots. The most prominent trend in Fig. 9 is that as pitch

Table 1 - GTM cases for "well converged" solutions with constant 36,000 total iterations. USM3D/SA, $f=0.43, \quad 0.12$, $0.05 \mathrm{~Hz}, \alpha_{0}=24 \mathrm{deg}, \Delta \alpha=+/-5 \mathrm{deg}$.

\begin{tabular}{|c|c|c|c|c|}
\hline & & \multicolumn{3}{|c|}{$\begin{array}{c}\text { Pitch freq, } \mathrm{Hz} \\
\text { (reduced frequency, } k \text { ) }\end{array}$} \\
\hline $\begin{array}{c}\text { Time- } \\
\text { step / } \\
\text { cycle }\end{array}$ & $\begin{array}{c}\text { Number } \\
\text { Sub iter }\end{array}$ & $\begin{array}{c}0.43 \\
(0.0144)\end{array}$ & $\begin{array}{c}0.12 \\
(0.0040)\end{array}$ & $\begin{array}{c}0.05 \\
(0.0017)\end{array}$ \\
\hline 360 & 100 & $360 / 100$ & $360 / 100$ & $360 / 100$ \\
\hline 720 & 50 & $720 / 50$ & $720 / 50$ & $720 / 50$ \\
\hline 1440 & 25 & $1440 / 25$ & $1440 / 25$ & $1440 / 25$ \\
\hline
\end{tabular}
frequency decreases (left to right), the order of magnitude residual drop increases from approximately -4 to the about -2.5 suggesting a less robust convergence, and the converged $\mathrm{CM}$ becomes more unsteady. At each frequency, however, the quality of the well-converged subiterative convergence is similar (top to bottom) regardless of the per-cycle [time step]/[subiteration] combination 360/100, $720 / 50$, or $1440 / 25$.

The dynamic pitching moment coefficients for the cases in Table 1 and Fig. 9 are plotted in Fig 10. The solutions exhibit almost no sensitivity of the $C_{m}$ hysteresis to these prescribed combinations of time step and sub-iteration, indicating that solution accuracy is predominantly a function of total iteration count. The general shapes of the $C_{m}$ hysteresis curves are remarkably similar between CFD and experiment. Recall from the discussion of Fig. 7 that the wind tunnel data was derived as an average or "mean" of highly unsteady dynamic measurements. This "mean" is similar to averaging that occurs due to the integration used with harmonic analysis methods to determine the inphase and out-of-phase dynamic stability derivatives [16]. The CFD is computed with the assumption of "Reynolds- 
Averaged Navier-Stokes" [19], which completely filters out any unsteady turbulent eddies in this massively separated post-stall flow. Thus, it is intriguing that these two fundamentally different approaches to averaging produce such similar results. Before pursuing more costly turbulence models that resolve turbulent eddies, such as Detached Eddy Simulation, further study of the URANS formulation might be warranted to explore its applicability and limitations for modeling post-stall dynamic stability characteristics.

\section{B. Searching for Efficient Solution Strategy}

Table 2 conveys the $f=0.43 \mathrm{~Hz}$ cases where subiterations are reduced consistently for each 360,720 , and 1440 time step strategy to maintain comparable levels of total iterations. The goal is to determine the least number of total iterations needed to maintain reasonably converged dynamic $C_{m}$. The blue highlighted cells from Table 1 are repeated in Table 2.

The convergence plots in Figure 11 are taken from the "well converged" solutions for $f=0.43 \mathrm{~Hz}$ in Figure 9 and 10 . Arrows at their respective positions on the convergence plots denote the reduced subiterations from Table 2 . The corresponding $C_{m}$ hysteresis loops are included in Fig. 11. From this, a visual correlation can be established between the subiterative convergence and the convergence of the $C_{m}$ hysteresis loops

Table 2 - GTM cases for determining the "most efficient" solution strategy. USM3D/ $\mathrm{SA}, f=0.43 \mathrm{~Hz}, \alpha_{0}=24 \mathrm{deg}, \Delta \alpha=+/-5 \mathrm{deg}$.

\begin{tabular}{|c|c|c|c|}
\hline $\begin{array}{r}\text { Iterations } \\
\text { per cycl }\end{array}$ & \multicolumn{3}{|c|}{$\begin{array}{c}\text { Time steps per } \\
\text { cycle/subiteration }\end{array}$} \\
\hline 36000 & $360 / 100$ & $720 / 50$ & $1440 / 25$ \\
\hline 18000 & $360 / 50$ & $720 / 25$ & $1440 / 13$ \\
\hline 14400 & $360 / 40$ & $720 / 20$ & $1440 / 10$ \\
\hline 10800 & $360 / 30$ & $720 / 15$ & $1440 / 8$ \\
\hline 7200 & $360 / 20$ & -------- & -------- \\
\hline 3600 & $360 / 10$ & --------- & -------- \\
\hline
\end{tabular}
relative to the "well converged" solution. It can be observed in Fig. 11(a) that the shape of the $C_{m}$ hysteresis changes from the "well converged" solution more rapidly below 30 subiterations with 360 steps per cycle. Hence reasonably converged dynamics solutions might be achieved on the GTM with as few as 10,800 total iterations per pitch cycle, which will be further tested for two lower frequencies in the next section using the highlighted cases (360/100,360/50, and 360/30) in Table 2.

The question of "how much accuracy is needed" remains unanswered and may be resolved using harmonic analysis and system identification techniques. These techniques add the requirement that sufficient sample rates be used to satisfy the Shannon Sampling Theorem. It is entirely possible that the accuracy with the 10 subiterations in Fig. 11(a) having 3600 total iterations per cycle is more than adequate for determining suitable dynamic stability derivatives for flight simulator models. The present study is primarily focused on establishing guidelines for numerical convergence.

\section{Testing Strategy at Varying Frequencies}

The next phase in the analysis involves testing the new strategy at lower oscillation frequencies of 0.12 , and $0.05 \mathrm{~Hz}$ to assess its validity at other dynamic conditions. The highlighted cells for $f=0.43 \mathrm{~Hz}$ from Table 2 are repeated in Table 3 . Results for the cases in Table 3 using less subiteration are plotted in Fig. 12 where small differences are noted in the $C_{m}$ hysteresis loops at the lower frequencies of 0.12 and $0.05 \mathrm{~Hz}$ as with the $f=0.43 \mathrm{~Hz}$ case. This confirms that the strategy of prescribing 360 steps per cycle and between 30 to 50 subiterations continues to hold in the low frequency range, although the results in Fig. 9

Table 3 - GTM cases to evaluate less subiteration at various frequencies. USM3D $/ \mathrm{SA}, \mathrm{f}=0.43,0.12,0.05 \mathrm{~Hz}, \alpha_{0}=24 \mathrm{deg}$, $\Delta \alpha=+/-5 \mathrm{deg}, 360$ steps per cycle.

\begin{tabular}{|c|c|c|c|c|}
\hline \multirow{2}{*}{ Strategy } & \multirow{2}{*}{ Total Iter } & \multicolumn{3}{|c|}{ Frequency, $\mathrm{Hz}$} \\
\cline { 3 - 5 } & & 0.43 & 0.12 & 0.05 \\
\hline "Well Converged" & 36000 & $360 / 100$ & $360 / 100$ & $360 / 100$ \\
\hline "Intermediate" & 18000 & $360 / 50$ & $360 / 50$ & $360 / 50$ \\
\hline "Most Efficient" & 10800 & $360 / 30$ & $360 / 30$ & $360 / 30$ \\
\hline
\end{tabular}
offer a warning of potential difficulties in converging dynamic solutions at lower frequencies. For this particular case, reasonably converged solutions can be obtained on the GTM across the desired frequency range with as few as 10800 total solution iterations per pitch cycle. Such computations require approximately $275 \mathrm{CPU}$ hours per cycle on an Intel Xeon ${ }^{\circledR} 3 \mathrm{GHz}$ cluster for the USM3D/SA code. 


\section{Testing Strategy for Advanced Turbulence Model}

The initial stages of the guideline study were conducted with the SA turbulence model. Similar computations are performed with the SST model to analyze impact of the strategy on a more advanced turbulence model. As noted in Table 4, the computations are performed for $\alpha_{0}=24 \mathrm{deg}$, $\Delta \alpha=+/-5 \mathrm{deg}$ across the full range of pitch frequencies $0.43,0.12$, and $0.05 \mathrm{~Hz}$ with 360 steps per cycle using 30 and 50 subiterations. The comparisons in Fig. 13 demonstrate that the variation from 30 to 50 subiterations does not significantly alter the behavior of the $C_{m}$ hysteresis for either of the turbulence models. Both turbulence models demonstrate the capacity

Table 4 - GTM cases to evaluate strategy on advanced SST turbulence model at various frequencies. $\mathrm{USM3D} /(\mathrm{SST}$ and SA), $\mathrm{f}=\mathbf{0 . 4 3}, 0.12,0.05 \mathrm{~Hz}, \alpha=24 \mathrm{deg}$, $\Delta \alpha=+/-5 \mathrm{deg}, 360$ steps per cycle.

\begin{tabular}{|c|r|r|r|}
\hline \multirow{2}{*}{$\begin{array}{c}\text { Turb Model } \\
\Delta \alpha=+/-5 \text { deg }\end{array}$} & \multicolumn{3}{|c|}{ Frequency, Hz } \\
\cline { 2 - 4 } & 0.43 & 0.12 & 0.05 \\
\hline \multirow{2}{*}{$\mathrm{SST}$} & $360 / 30$ & $360 / 30$ & $360 / 30$ \\
& $360 / 50$ & $360 / 50$ & $360 / 50$ \\
\hline \multirow{2}{*}{$\mathrm{SA}$} & $360 / 30$ & $360 / 30$ & $360 / 30$ \\
& $360 / 50$ & $360 / 50$ & $360 / 50$ \\
\hline
\end{tabular}
to capture the general behavior of $C_{m}$ hysteresis for the GTM at this post-stall condition. While some changes of shape in the $C_{m}$ hysteresis are apparent between the SST and SA models, the primary difference is an offset in $C_{m}$ levels that is consistent with the offset in static $C_{m}$ in Fig. 6 at $\alpha=24$ deg. These results highlight the strong impact of turbulence model on the post-stall solution that must be considered carefully.

\section{E. Evaluation for Larger Pitch Amplitude}

The devised strategy is next evaluated for a more challenging dynamic condition with larger pitch amplitude $\Delta \alpha=+/-10$ deg using 360 time steps per cycle. The larger of the two prescribed subiterations in Table 5 is set to 100 to insure a well-converged reference condition for this more difficult condition. Both the SA and SST turbulence models are also retained for the comparisons. The computations presented in Fig. 14 demonstrate that the SA and SST solutions each capture the general size of the experimental $C_{m}$ hysteresis loops across the frequency range. Some differences are apparent in the details of the shapes. However as discussed earlier, the "mean" wind tunnel data is extracted

Table 5 - GTM cases to evaluate strategy on larger pitch amplitude. USM3D/(SST and SA), $\mathrm{f}=0.43,0.12$, $0.05 \mathrm{~Hz}, \alpha=24 \mathrm{deg}, \Delta \alpha=+/-10 \mathrm{deg}, 360$ steps per cycle.

\begin{tabular}{|c|c|c|c|}
\hline \multirow{2}{*}{$\begin{array}{c}\text { Turb Model } \\
\Delta \alpha=+/-10 \text { deg }\end{array}$} & \multicolumn{3}{|c|}{ Frequency, Hz } \\
\cline { 2 - 4 } & 0.43 & 0.12 & 0.05 \\
\hline \multirow{2}{*}{ SST } & $360 / 30$ & $360 / 30$ & $360 / 30$ \\
& $360 / 100$ & $360 / 100$ & $360 / 100$ \\
\hline \multirow{2}{*}{ SA } & $360 / 30$ & $360 / 30$ & $360 / 30$ \\
& $360 / 100$ & $360 / 100$ & $360 / 100$ \\
\hline
\end{tabular}
from highly unsteady dynamic measurements and its qualitative similarity with the URANS CFD result is remarkable. The actual "goodness" or lack thereof of the computational results remains an open question to be resolved.

\section{F. Evaluation for Grid Sensitivities}

Finally, an assessment of grid sensitivities on the periodic oscillating solution is provided using the two finer grids having 12- and 24-million cells. The computations were performed with the USM3D/SA model at $f=0.43 \mathrm{~Hz}$, $\alpha_{0}=24 \mathrm{deg}, \Delta \alpha=+/-5 \mathrm{deg}$ using 360 steps per cycle and 100 subiterations. As observed in Fig. 15, the general shape of the $C_{m}$ hysteresis is maintained for the three grids at $f=0.43 \mathrm{~Hz}$, but tend toward collapse at the extrema for the two lower frequencies of 0.12 and $0.05 \mathrm{~Hz}$. The overall reduced vertical dimensions of the ellipses properly reflect the reduced damping force as frequency (and corresponding maximum pitch rates) is reduced. The small differences in $C_{m}$ offset are consistent with the offset of static $C_{m}$ in Fig. 5 at $\alpha=24$ deg. In comparison to the analysis from the preceding two sections, these results demonstrate that the choice of turbulence model has far more impact on solution correlation than the grid density. Hence as a general rule, numerical guidelines can be developed on a relatively coarse grid for this class of problem, as long as it has sufficient resolution to produce static solutions to a reasonably level of accuracy. 


\section{Preliminary Harmonic Analysis}

Harmonic analysis offers a common framework to compare dynamic models from two independent modeling methodologies. Figures 16 compares linear harmonic models estimated from both wind tunnel and CFD time histories. In addition, wind tunnel measurements, mean wind tunnel measurements, and USM3D/SA predictions are shown. Three different frequencies of oscillation, at $0.43,0.12$, and $0.05 \mathrm{~Hz}$, with nominal oscillations about $\alpha_{0}=24$ degrees and with $\Delta \alpha=+/-5$ degrees were analyzed. In this high angle of attack regime, well beyond stall, nonlinear and unsteady behaviors are expected, however the relatively small amplitude of oscillation limits the degree of nonlinear responses.

The deviation of measured and computed hysteresis loops from the $1^{\text {st }}$ harmonic ellipses (dashed lines) in Fig. 16 reflects the degree of aerodynamic nonlinearity. Consequently, the regular ellipses formed by the linear harmonic models represent the best fit to the nonlinear responses shown. Excluding bias errors (that reflect static model differences) between the two harmonic models, the differences in loop size at the same oscillation frequency reflect the differences in the out-of-phase coefficients. Overall matching of the harmonic model responses from the two independent modeling methodologies is encouraging and supports further investigation.

In-phase and out-of-phase coefficient estimates from this analysis were consistent between the two modeling methodologies. Out-of-phase coefficients estimated from wind tunnel and USM3D data both reflect a clear dependence on frequency and therefore the presence of unsteady behaviors. Differences in coefficient values, averaged over 3 frequencies, were $18 \%$ for $C_{N q}$ and $42 \%$ for $C_{m q}$. In-phase coefficients from both sources were also consistent and demonstrated negligible frequency dependence. Differences in these coefficient values averaged $1 \%$ for $C_{N_{\alpha}}$ and $7 \%$ for $C_{m_{\alpha}}$. In-phase coefficient values are comparable with the static data slopes. Observing a larger difference for both harmonic models in estimating out-of-phase coefficients compared to in-phase coefficients is consistent with general aircraft identification experience in estimating static versus dynamic terms.

Modeling dynamic systems adds a requirement that sufficient sample rates be used that satisfy the Shannon Sampling Theorem. Wind tunnel measurements were made with a sample rate of $250 \mathrm{~Hz}$, an anti-alias filter at 100 $\mathrm{Hz}$, and a final low-pass digital filter at $4 \mathrm{~Hz}$. In this study, sample rates in Hertz for the CFD data were dependent upon the product of oscillation frequency and samples/cycle. Consequently, the lowest sample rates of $18 \mathrm{~Hz}$ occurred for the lowest frequency cases. For this particular study, aircraft configuration, and conditions considered, sample rates of approximately $18 \mathrm{~Hz}$ may be sufficient for the CFD methodology.

\section{Summary}

A systematic study has been presented using the NASA Generic Transport Model to guide the selection of a numerical solution strategy for URANS computation of a subsonic transport configuration undergoing simulated forced oscillation about its pitch axis. Forced oscillation is central to the prevalent wind tunnel methodology for quantifying aircraft dynamic stability derivatives from force and moment coefficients, which is the ultimate goal for the computational simulations. A preliminary linear harmonic analysis was also performed to demonstrate the potential of extracting reasonable values for dynamic stability derivatives from computational solutions.

Several key insights can be summarized from the preceding analysis. The following assumes the dynamic simulations are performed with a URANS flow solver and turbulence model using a $2^{\text {nd }}$-order time stepping scheme, and low-speed flow on a subsonic transport configuration. Among the observations noted from the GTM study are:

- Convergence of periodic oscillating solutions is a function of the total numbers of solution iterations. Figures 9 and 10 confirm that for any angle of attack the dynamic solutions converge to the same answer regardless of the path as long as (total solution iterations per cycle $)=($ number of time steps per cycle)*(number of subiterations) remain constant. There are apparently no shortcuts to convergence. Hence in future attempts to establish time step and subiteration guidelines, there is no need to run a wide range of time steps, as in Table 1, for the purpose of determining solution convergence. One carefully selected case should be adequate for determining a solution strategy.

- Dynamic solutions may be more difficult to converge at the lower oscillation frequencies. Subiterative solution convergence between time steps $n$ and $n+1$ at the lower frequencies of 0.12 and $0.05 \mathrm{~Hz}$ during the GTM forced pitch oscillation cycle exhibit reluctant numerical convergence and an unsteady behavior in Fig. 9. This suggests a stronger potential to encounter convergence difficulties at lower frequencies than at higher ones. Hence, one should consider starting a convergence guideline study by focusing on a "potentially difficult" case at the lowest frequency of interest so that the resulting guidelines should readily apply at the better-behaved higher frequencies. 
- A dynamic convergence study can be performed on a reasonably coarse grid to conserve resources. Once a solution strategy has been identified, sensitivities to grid resolution should be investigated further with the determined strategy.

- For forced oscillation problems in regions of post-stall flow, the selection of turbulence model has far more impact than grid resolution. Ref. [6] demonstrates how the static force and moment coefficients can provide useful guidance for dynamic studies. The single $\alpha_{0}=24$ deg condition was selected for the present study from Fig. 5 as representative of a post-stall flight condition.

- Simulations of forced oscillation in post-stall flows with the URANS formulation yields remarkable correlation with the time-averaged "mean" of highly unsteady dynamic wind tunnel data. The URANS formulation assumes Reynolds averaging of the Navier-Stokes equations that effectively filters out any unsteady turbulent eddies from massively separated flows. Yet, the overall size and shape of the computed $C_{m}$ hysteresis are very similar to the time-averaged "mean" of highly unsteady forcedoscillation wind tunnel data.

- The conclusions from this analysis of a subsonic transport configuration also apply to a fundamentally different configuration and flow field. Each of the preceding observations is fully consistent with those for a different class of unmanned air combat vehicle dominated by vortical flow in Ref. [6].

- The question of "how accurate do dynamic CFD solutions have to be" remains unanswered. Since the final product of CFD are dynamic stability derivatives for flight simulator models, further analysis of the URANS solutions using harmonic analysis and system identification techniques is warranted before pursuing more costly high-resolution turbulence models, such as Detached Eddy Simulation. A preliminary harmonic analysis of the GTM solutions suggests that linear out-of-phase dynamic stability derivatives can be extracted from CFD within 18- to 42-percent of experimentally derived linear derivatives.

- Largest differences between wind tunnel and CFD harmonic models were observed for pitching moment models at lower frequencies. Lower frequencies present a more difficult estimation problem for both wind tunnel and CFD cases since the damping forces are smaller leading to reduced signal/noise ratio for identification. Future identification work should include confidence interval estimation for both CFD and wind tunnel parameter estimates that will allow better accuracy comparisons.

- Frequency dependence was observed in the CFD out-of-phase coefficients. This result reflects the presence of unsteady behaviors and is consistent with that obtained from the wind tunnel estimates. Overall consistency between the two methodologies in predicting dynamic response supports further investigation into applying CFD methods to difficult stability and control modeling problems.

- Broader input spectrums for both the CFD and wind tunnel testing methodologies are desirable. Additional frequencies of sinusoidal forced oscillations tests would allow estimation of more general dynamic models. However, advanced identification inputs such wide-band inputs or arbitrary motion would allow more efficient estimation of general mathematical models that predict nonlinear responses and unsteady aerodynamic lags.

\section{Acknowledgments}

The authors gratefully acknowledge the generous support for this work from the NASA Aviation Safety Program, Integrated Resilient Aircraft Controls (IRAC) project, and the NASA Engineering and Safety Office (NESC).

\section{References}

1. Cunningham, Kevin, Foster, John V., Shah, Gautam H., Stewart, Eric C., Ventura, Robin N. (2005), "Simulation Study of Flap Effects on a Commercial Transport Airplane in Upset Conditions", AIAA 2005-5908

2. Foster, J.V., Cunningham, K., Shah, G.H., Stewart, E.C., Ventura, R.N. (2005), "Dynamic Modeling and Simulation of Large Transport Airplanes in Upset Conditions", AIAA 2005-5933, August 2005.

3. Gortz, Stefan, McDaniel, David R. (2007), "Towards and Efficient Aircraft Stability and Control Analysis Capability using High-Fidelity CFD”, AIAA 2007-1053

4. Kimberlin, R. D., "Flight Testing of Fixed-Wing Aircraft", AIAA Educational Series, 2003. 
5. Frink, N.T., Pirzadeh, S.Z., Atkins, H.L., Viken, S.A., and Morrison, J.H., "CFD Assessment of Aerodynamic Degradation of a Subsonic Transport Due to Airframe Damage", AIAA 2010-500, January 2010.

6. Frink, N.T., "Strategy for Dynamic CFD Simulations on SACCON Configuration”, AIAA 2010-4559, June 2010.

7. Shah, Gautam H.; Cunningham, Kevin; Foster, John V.; Fremaux, C. Michael; Stewart, Eric C.; Wilborn, James E.; Gato, William; and Pratt, Derek W.: "Wind Tunnel Investigation of Commercial Transport Aircraft Aerodynamics at Extreme Flight Conditions”, SAE-2002-01-2912, November 2002.

8. Brandon, Jay M., et.al. "Comparison of Rolling Moment Characteristics During Roll Oscillations for a Low and High Aspect Ratio Configuration," AIAA Atmospheric Flight Mechanics Conference, AIAA 2004-5273, AIAA Washington, DC, 2004.

9. Murphy, P. C. and Klein, V.: "Transport Aircraft System Identification from Wind Tunnel Data," AIAA Atmospheric Flight Mechanics Conference, AIAA 2008-6202, AIAA Washington, DC, 2008.

10. Braslow, A.L. Hicks, R.M., and Harris, Jr., R.V.: "Use of Grit-Type Boundary Layer-Transition Trips on Wind Tunnel Models", NASA TN D-3579, September 1966.

11. Frink, N. T., "Tetrahedral Unstructured Navier-Stokes Method for Turbulent Flows," AIAA Journal, Vol. 36, No. 11, November 1998, pp. 1975-1982.

12. Frink, N. T., Pirzadeh, S. Z., Parikh, P. C., Pandya, M. J.(2000) The NASA Tetrahedral Unstructured Software System. Aeronautical Journal, Vol. 104, No. 1040 (491-499)

13. Pandya, M.J., Frink, N.T., Abdol-Hamid, K.S., and Chung, J.J.: "Recent Enhancements to USM3D Unstructured Flow Solver for Unsteady Flows", AIAA 2004-5201, August 2004.

14. Pirzadeh, S. Z., "Advanced Unstructured Grid Generation for Complex Aerodynamic Applications," AIAA Journal, Vol. 48, No. 5, pp. 904-915, 2010.

15. Pirzadeh, S. Z., "Unstructured Viscous Grid Generation by the Advancing-Layers Method," AIAA Journal, Vol. 32, No. 8, pp. 1735-1737, 1994.

16. Kim, S., Murphy, P. C. and Klein, V.: "Evaluation and Analysis of F-16XL Wind Tunnel Data From Dynamic Tests," AIAA Atmospheric Flight Mechanics Conference, AIAA 2003-5396, AIAA Washington, DC, 2010.

17. Klein, Vladislav, Murphy, Patrick C., and Szyba, Nathan M., "Analysis of Wind Tunnel Oscillatory Data of the F-16XL Aircraft," NASA TM-2004-213246, August, 2004.

18. Roe, P. L., "Approximate Riemann Solvers, Parameter Vectors, and Difference Schemes," Journal of Computational Physics, Vol. 43, 1981, pp. 357-372.

19. Wilcox, D.C., Turbulence Modeling for CFD, $3^{\text {rd }}$ ed., DCW Industries, Inc., La Canada, California, 2006, Chap. 2.
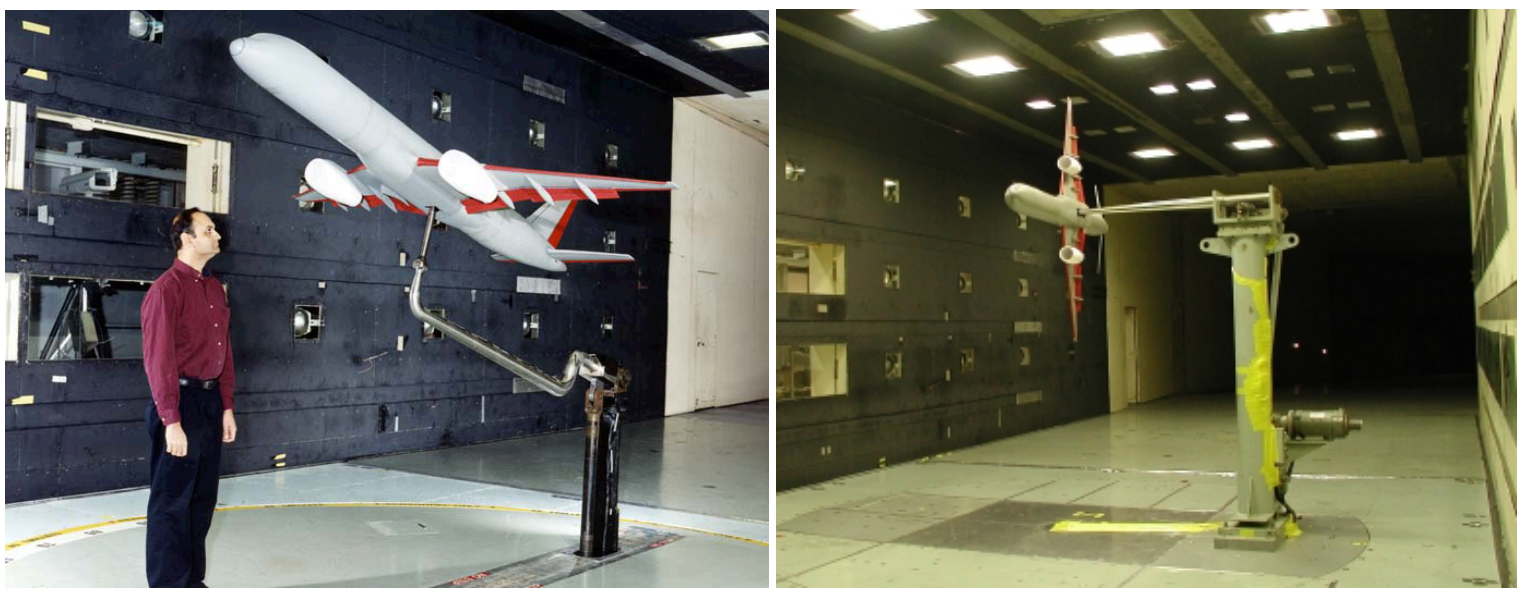

Figure 3. Generic Transport Model (GTM) in the NASA Langley 14- by 22-Foot Low-Speed Tunnel. Left static mount, Right - pitch oscillation mount. 


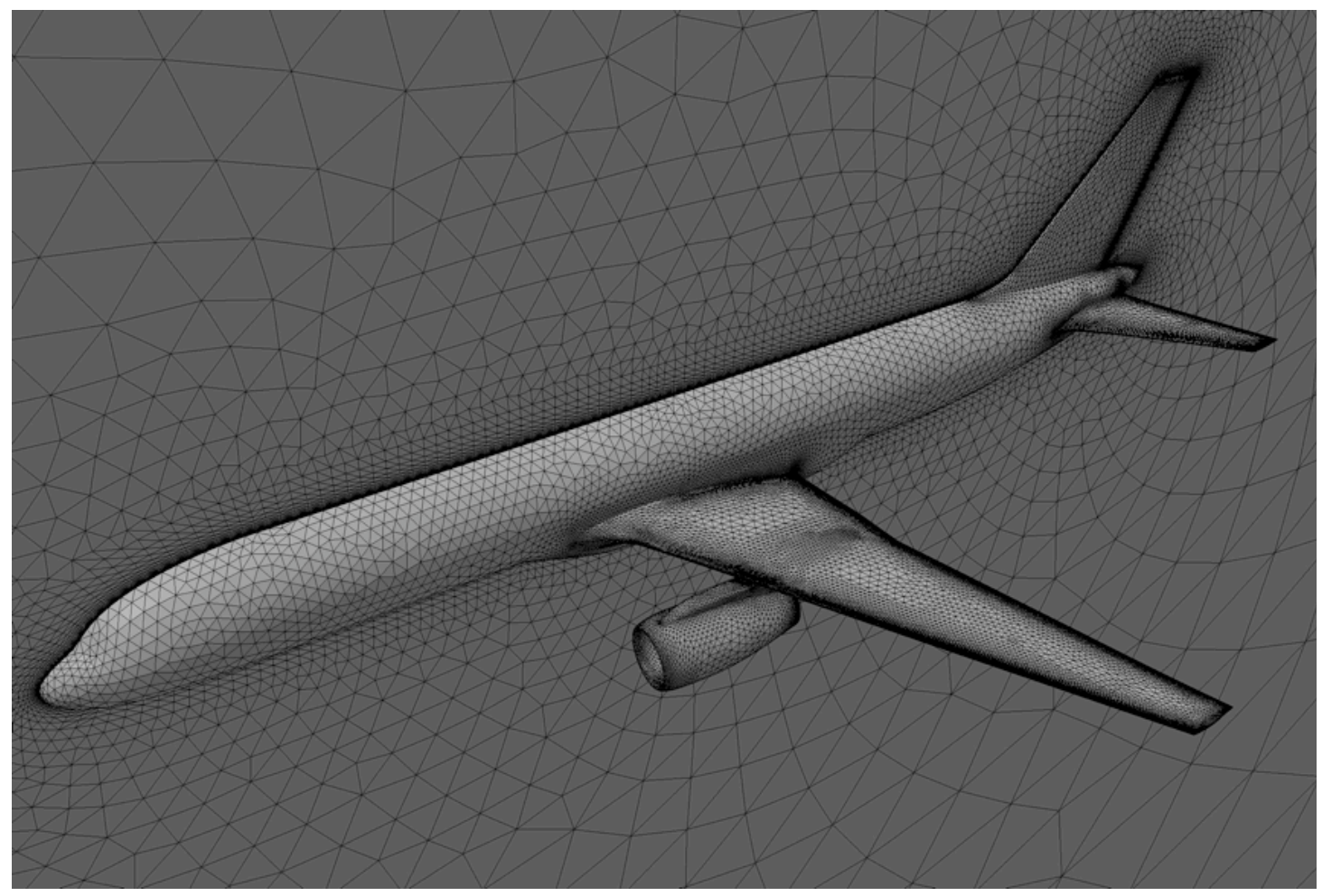

Figure 4. - Surface triangulation for half-span GTM. Volume contains 6-million tetrahedral cells.
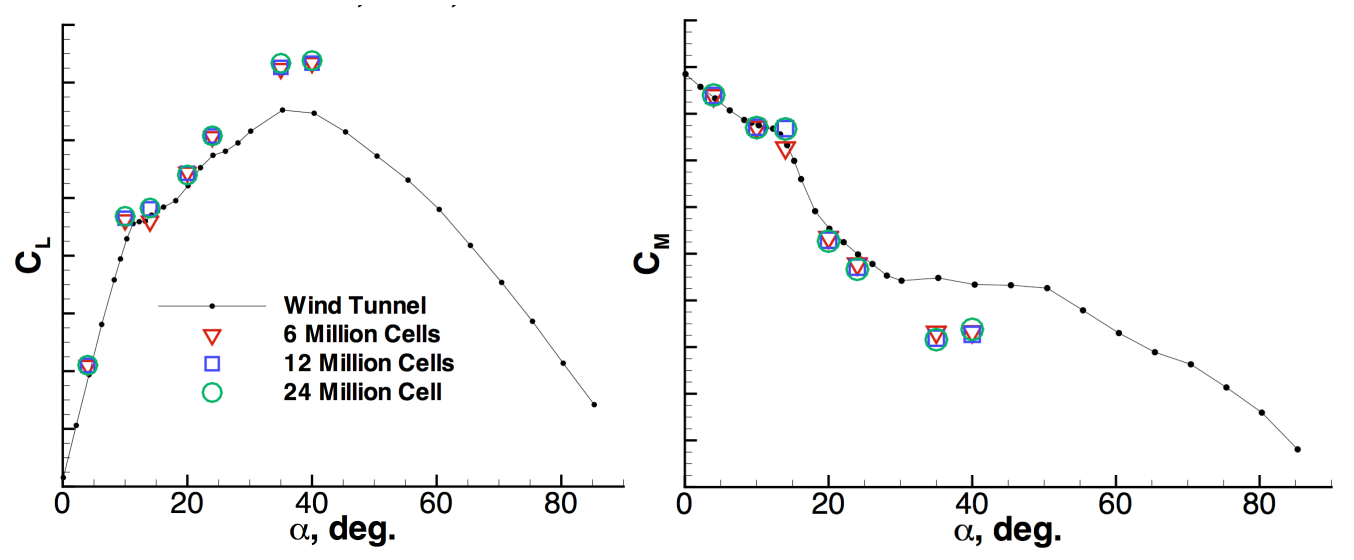

Figure 5. Effect of GTM grid refinement on static lift and pitching moment coefficient. $14 X 22 M_{\infty}=0.077$, USM3D/SA $M_{U S M 3 D}=0.2, \operatorname{Re}_{\text {cref }}=0.54 \times 10^{6}$. 

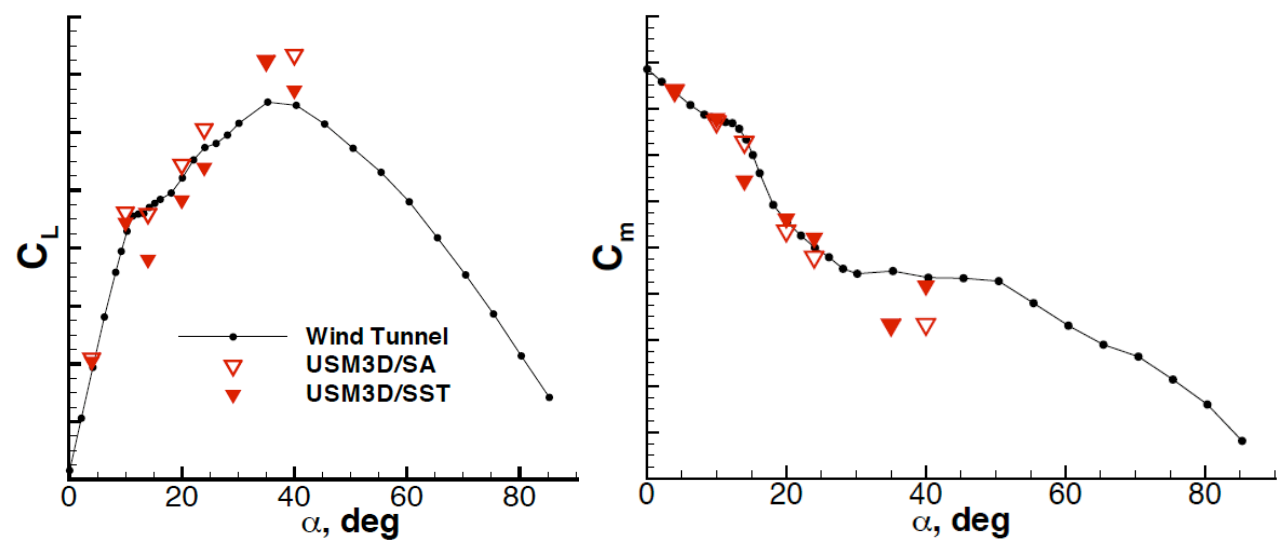

Figure 6. Effect of turbulence model on static lift and pitching moment coefficient on 6-million cell grid. $14 X 22 M_{\infty}=0.077$, USM3D/SA $M_{U S M 3 D}=0.2, R e_{c r e f}=0.54 \times 10^{6}$.
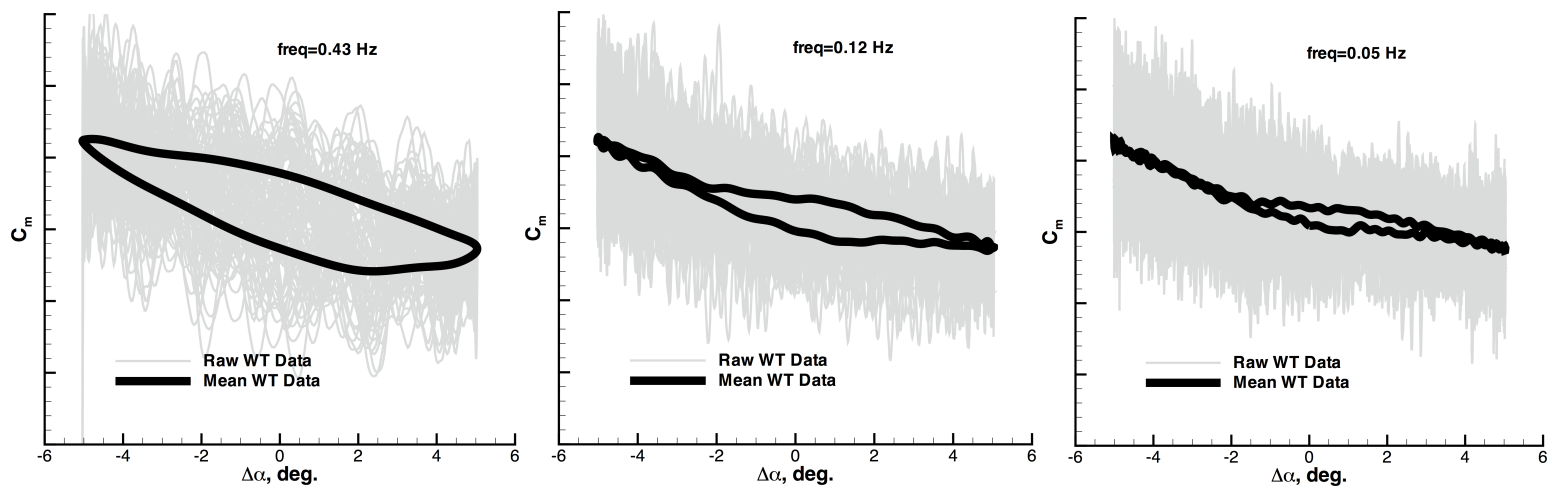

Figure 7. Raw and mean dynamic pitching moment coefficients from $14 X 22$ wind tunnel. $M_{\infty}=0.077$, $\operatorname{Re}_{\text {cref }}=0.54 \times 10^{6}, \alpha_{0}=24 \mathrm{deg}, \Delta \alpha=+/-5 \mathrm{deg}$.

USM3D time-accurate flow solution with pitch oscillation of 5 deg

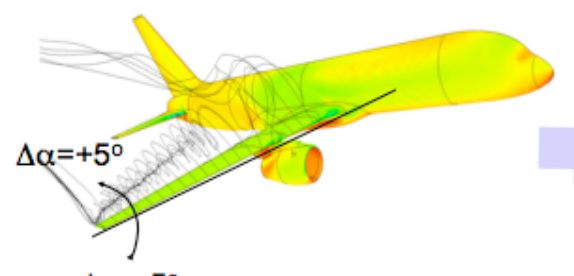

$\Delta \alpha=-5^{\circ}$
Pitching Moment Hysteresis

Plot

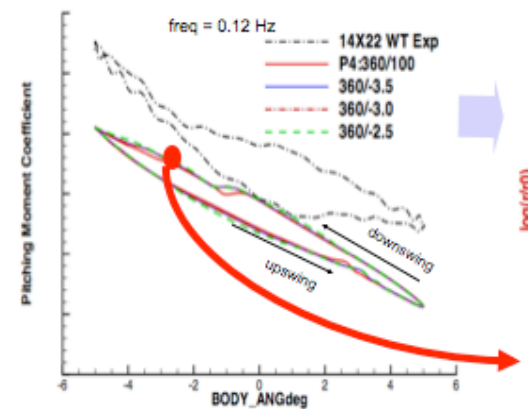

Subiterative Convergence of Pitching Moment at $\Delta \alpha=-3.5$ degrees

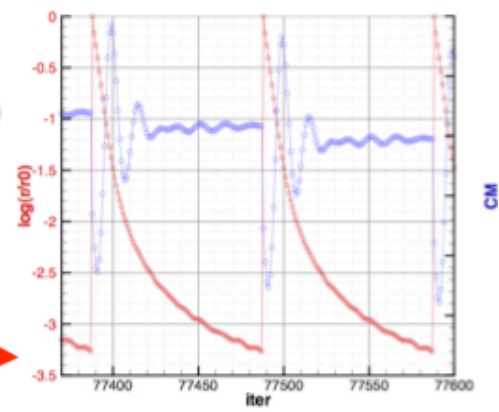

Figure 8. Approach for establishing visual correlation between time step and subiterative convergence for GTM undergoing pitch oscillation. 

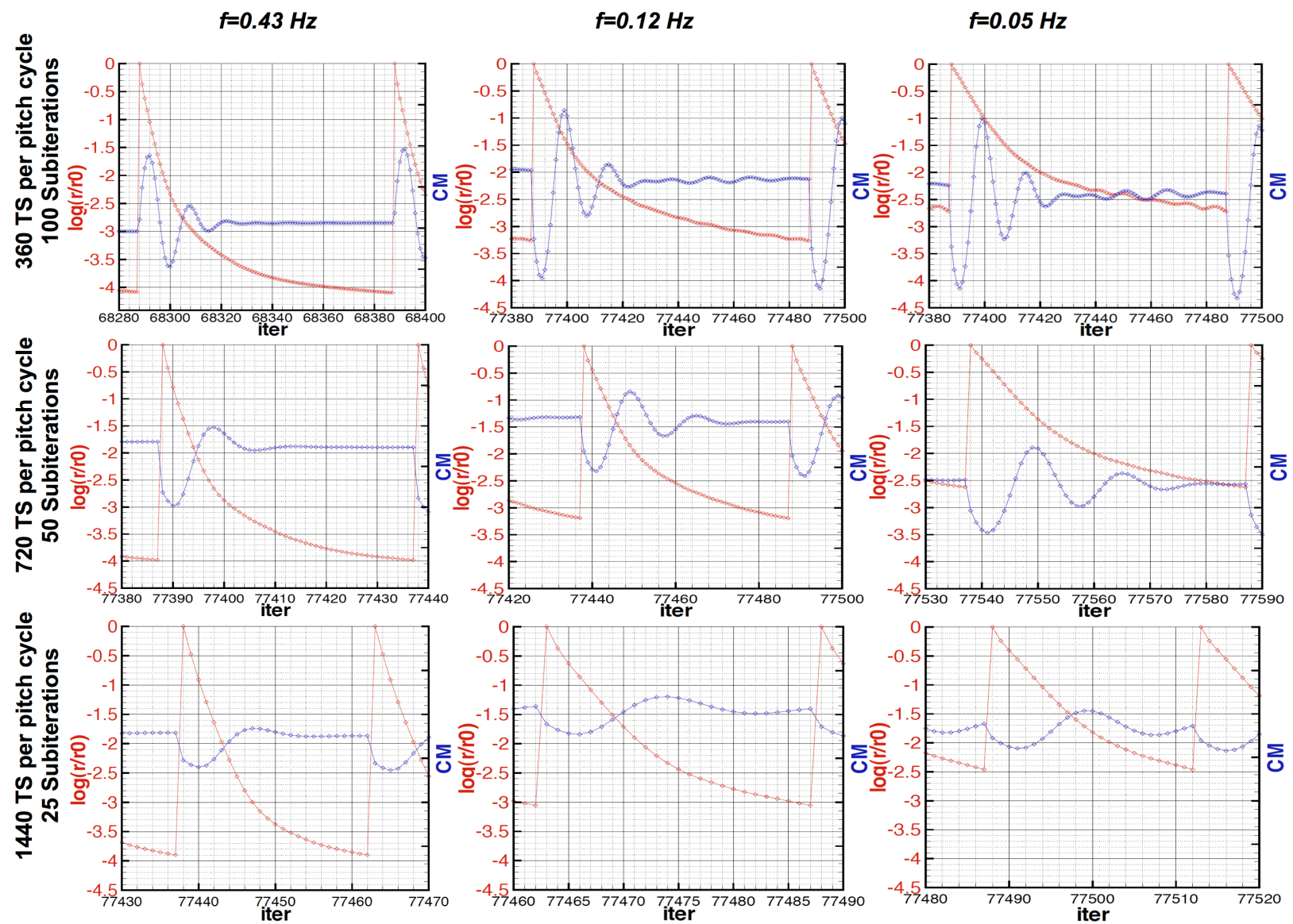

Figure 9. Correlation of number of $2^{\text {nd }}$-order time steps with subiterations on convergence at downswing $\Delta \alpha=-3.5 \mathrm{deg}$ for GTM. Constant 36,000 total iterations maintained. USM3D/SA $M_{U S M 3 D}=0.2$, $\operatorname{Re}_{\text {cref }}=0.54 \times 10^{6}, \alpha_{0}=24 \mathrm{deg}, \Delta \alpha=+/-5 \mathrm{deg}$.
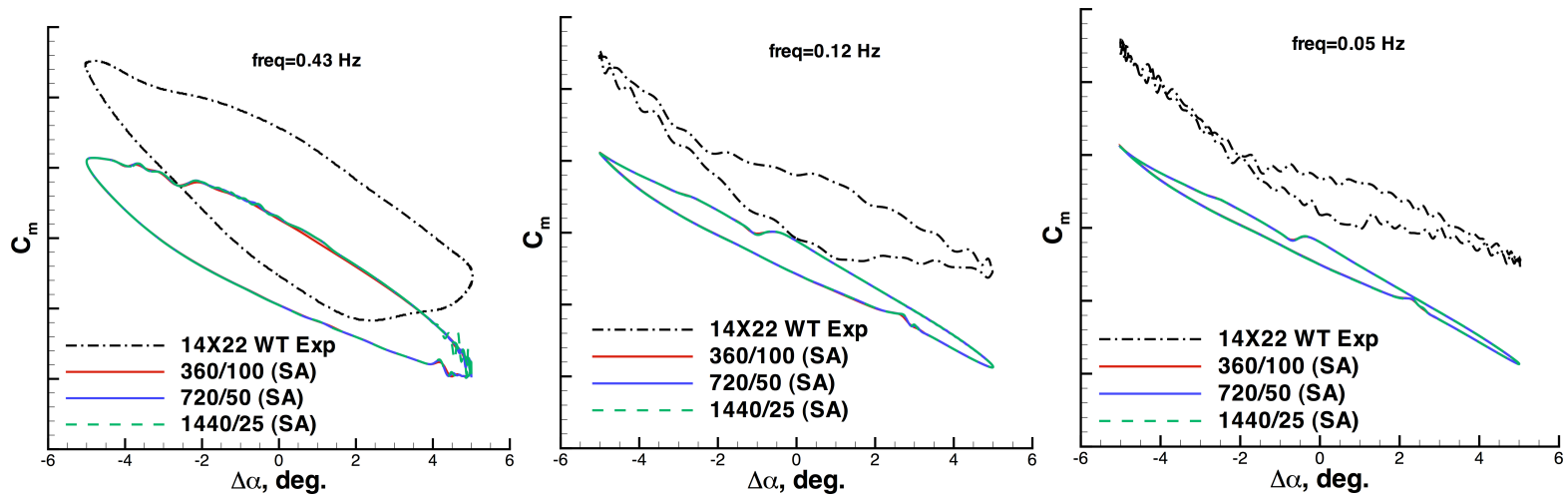

Figure 10. The effects of combined (time-step)*(subiteration) $=36000$ iterations on GTM dynamic pitching moment coefficients. $14 X 22 M_{\infty}=0.077$, USM3D/SA $M_{U S M 3 D}=0.2, R e_{c r e f}=0.54 \times 10^{6}, \alpha_{0}=24 \mathrm{deg}, \Delta \alpha=+/-5 \mathrm{deg}$. L-R: Frequencies of $0.43,0.12,0.05 \mathrm{~Hz}$ 

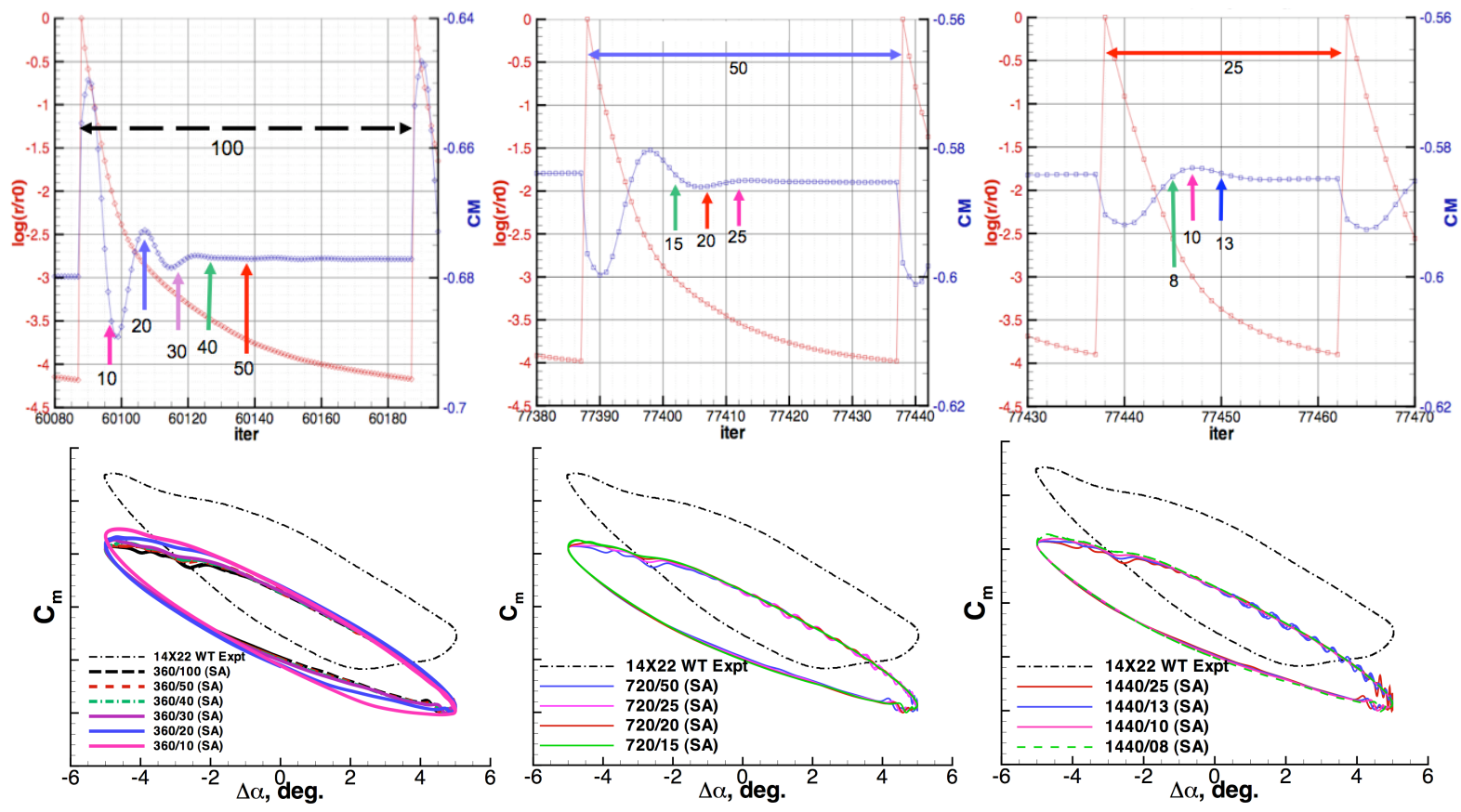

Figure 11. - Convergence correlations of less subiteration wth GTM dynamic pitching moment coefficient at $f=0.43$ Hz. $14 X 22 M_{\infty}=0.077$, USM3D/SA $M_{U S M 3 D}=0.2, R e_{c r e f}=0.54 \times 10^{6}, \alpha_{0}=24$ deg, $\Delta \alpha=+/-5$ deg. L-R: Number of steps per cycle a) 360, b) 720, and c) 14400
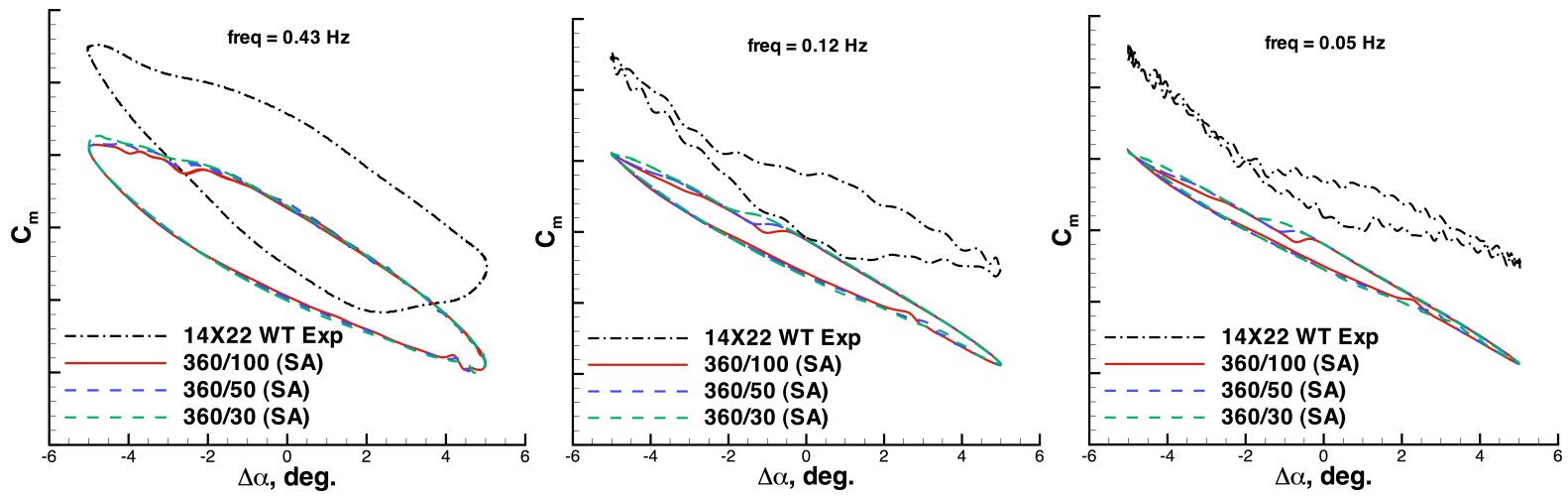

Figure 12.- Effect of less subiteration across GTM dynamic frequency range using 360 time steps per cycle. $14 X 22 M_{\infty}=0.077$, USM3D/SA $M_{U S M 3 D}=0.2, R e_{c r e f}=0.54 \times 10^{6}, \alpha_{0}=24 \mathrm{deg}, \Delta \alpha=+/-5 \mathrm{deg}$. L-R: Frequencies a) $0.43 \mathrm{~Hz}$, b) $0.12 \mathrm{~Hz}$, and c) $0.05 \mathrm{~Hz}$. 

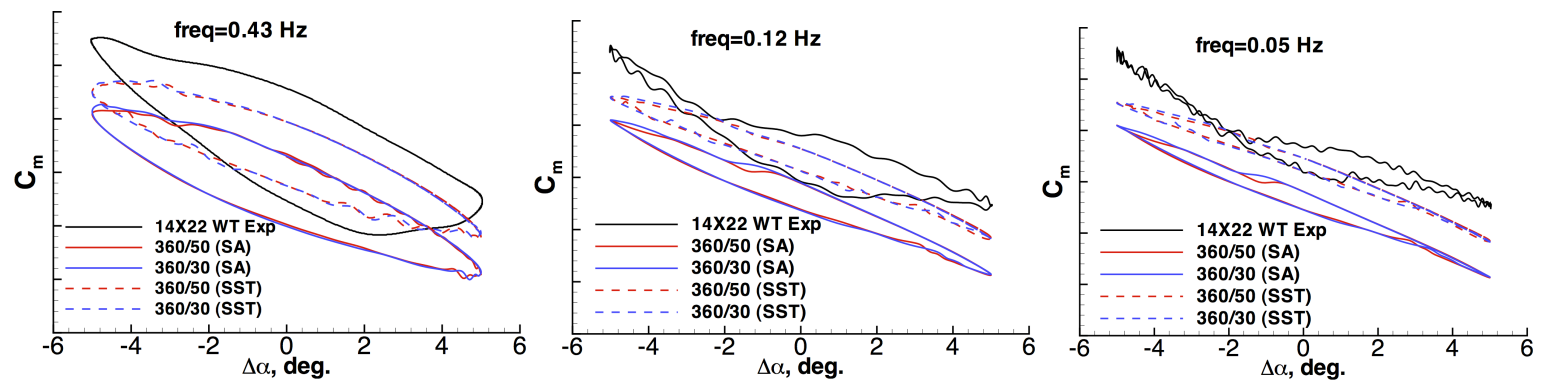

Figure 13. Effect of less subiteration on GTM with advanced SST turbulence model using 360 time steps per cycle. $14 X 22 M_{\infty}=0.077$, USM3D/(SA and SST) $M_{U S M 3 D}=0.2, R e_{\text {cref }}=0.54 \times 10^{6}, \alpha_{0}=24 \mathrm{deg}, \Delta \alpha=+/-5 \mathrm{deg}$. L-R: Frequencies a) $0.43 \mathrm{~Hz}$, b) $0.12 \mathrm{~Hz}$, and c) $0.05 \mathrm{~Hz}$.
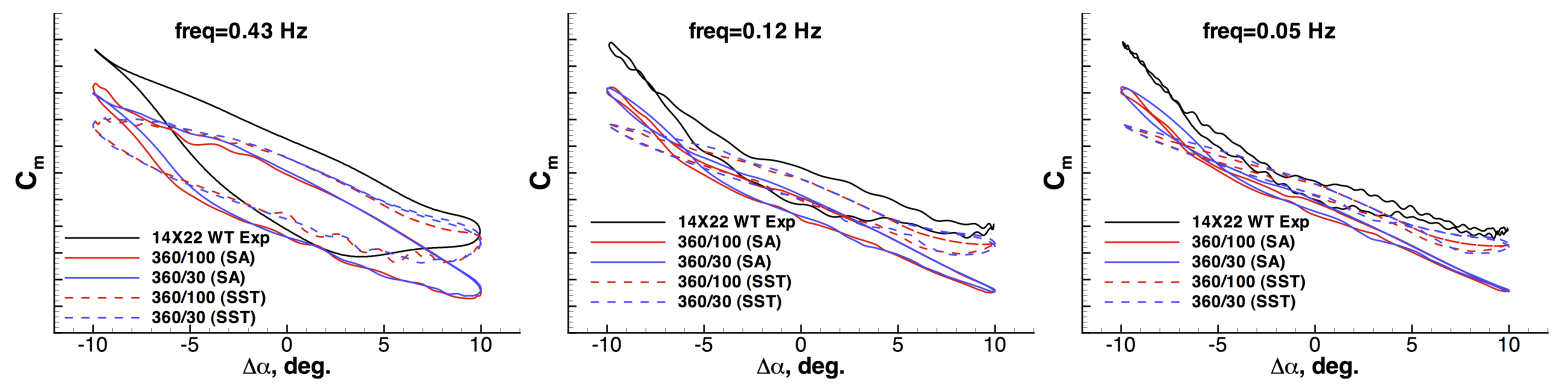

Figure 14. Effect of subiteration on GTM with larger amplitude of pitch oscillation $\left(\alpha_{0}=24 \mathrm{deg}, \Delta \alpha=+/-10\right.$ deg) using 360 time steps per cycle. $14 X 22 M_{\infty}=0.077, \mathrm{USM} 3 \mathrm{D} /\left(\mathrm{SA}\right.$ and SST) $M_{U S M 3 D}=0.2, R e_{\text {cref }}=0.54 \times 10^{6}$, L-R: Frequencies a) $0.43 \mathrm{~Hz}$, b) $0.12 \mathrm{~Hz}$, and c) $0.05 \mathrm{~Hz}$.
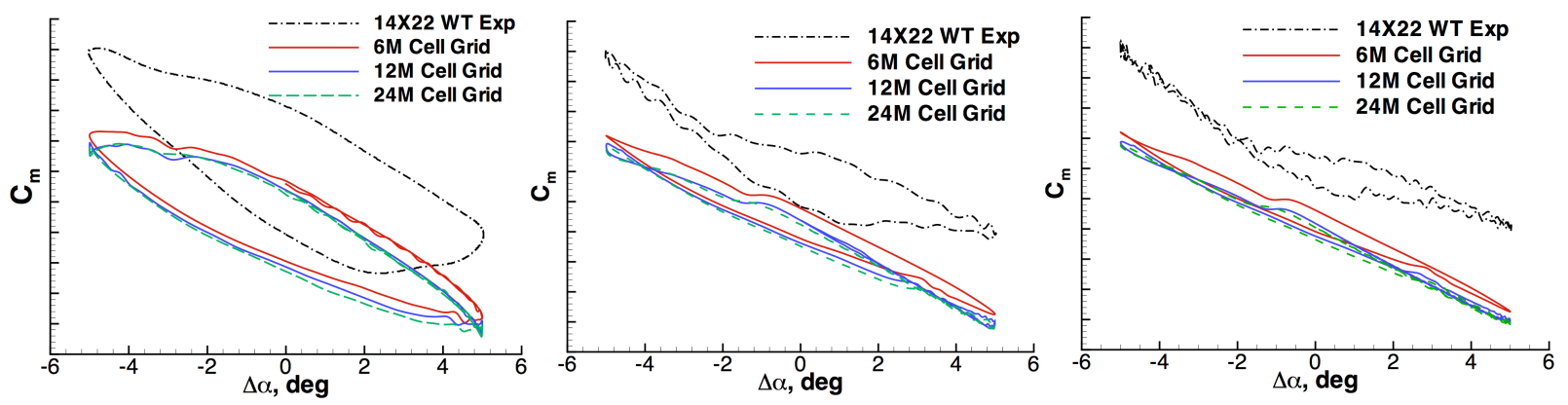

Figure 15. Effect of grid density on GTM dynamic pitching moment coefficient using 360 time steps per cycle, 100 subiterations. $14 X 22 M_{\infty}=0.077$, USM3D/SA $M_{U S M 3 D}=0.2, R e_{c r e f}=0.54 \times 10^{6}, \alpha_{0}=24 \mathrm{deg}, \Delta \alpha=+/-5 \mathrm{deg}$. L-R: 6-million, 12-million, and 24-million cells for half-span. 

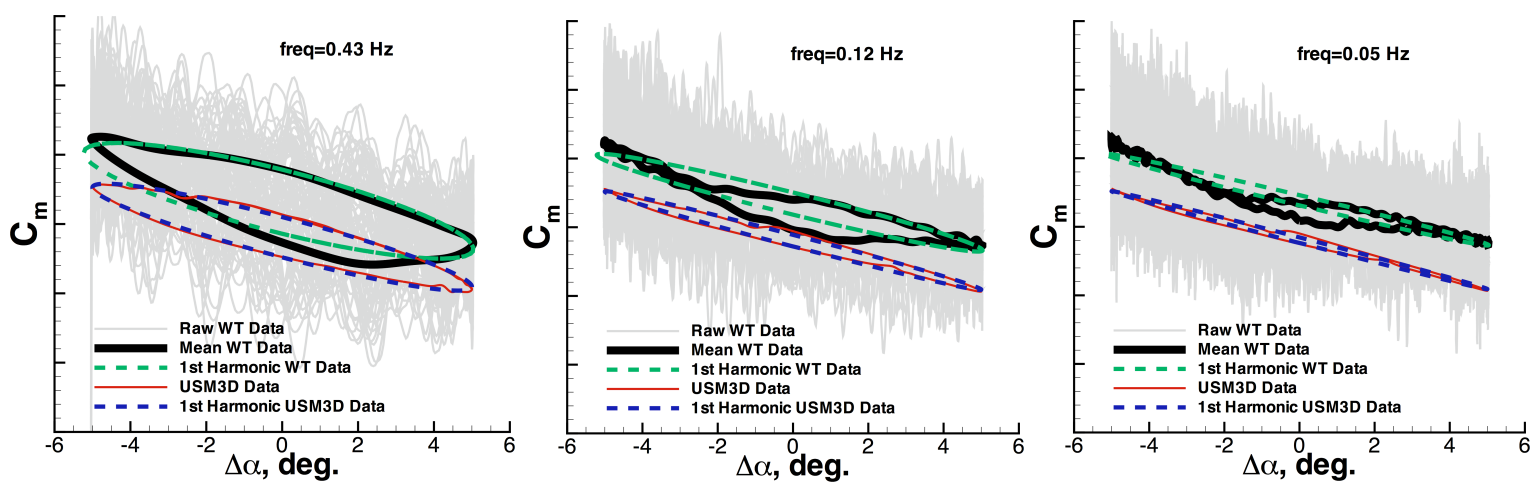

Figure 16. Preliminary harmonic analysis on dynamic pitching moment coefficient. 14X22 $M_{\infty}=0.077$, USM3D/SA $M_{U S M 3 D}=0.2,360$ steps per cycle, 100 subiterations, $R e_{c r e f}=0.54 \times 10^{6}, \alpha_{0}=24 \mathrm{deg}, \Delta \alpha=+/-5 \mathrm{deg}$. LR: Frequencies a) $0.43 \mathrm{~Hz}$, b) $0.12 \mathrm{~Hz}$, and c) $0.05 \mathrm{~Hz}$. 